\title{
HOW DO DRIVERS BEHAVE IN A HIGHLY AUTOMATED CAR?
}

\author{
Natasha Merat and A. Hamish Jamson \\ Institute for Transport Studies \\ University of Leeds \\ Leeds, UK \\ E-mail: N.Merat@its.leeds.ac.uk
}

\begin{abstract}
Summary: This paper outlines the results of a driving simulator study conducted for the European CityMobil project, which was designed to investigate the effect of a highly automated driving scenario on driver behaviour. Drivers' response to a number of 'critical' scenarios was compared in manual driving with that in automated driving. Drivers were in full control of the vehicle and its manoeuvres in the manual driving condition, whilst control of the vehicle was transferred to an 'automated system' in the automated driving condition. Automated driving involved the engagement of lateral and longitudinal controllers, which kept the vehicle in the centre of the lane and at a speed of $40 \mathrm{mph}$, respectively. Drivers were required to regain control of the driving task if the automated system was unable to handle a critical situation. An auditory alarm forewarned drivers of an imminent collision in such critical situations. Drivers' response to all critical events was found to be much later in the automated driving condition, compared to manual driving. This is thought to be because drivers' situation awareness was reduced during automated driving, with response only produced after drivers heard the alarm. Alternatively, drivers may have relied too heavily on the system, waiting for the auditory alarm before responding in a critical situation. These results suggest that action must be taken when implementing fully automated driving to ensure that the driver is kept in the loop at all times and is able to respond in time and appropriately during critical situations.
\end{abstract}

\section{OBJECTIVES}

This paper presents the results of one of the studies conducted for the EU funded project CityMobil, the main aim of which has been to introduce advanced urban transport systems on a large scale. One objective of CityMobil has been to investigate the human factors issues associated with future developments in urban public transport, where the driving task may become much more automated. The experiment described here investigated some of the issues associated with 'dual-mode' driving, where a particular vehicle can either be driven in manual mode (with all aspects of driving controlled by the driver) or in an automated mode, where the vehicle is effectively driven by means of various automated systems which control its longitudinal and lateral position within the road and with respect to other traffic. Such dual-mode vehicles can be driven on equipped 'eLanes' (Flemisch, 2005; Schieben et al., 2008) and consist of longitudinal and lateral guidance systems, radars for obstacle detection, vehicle to infrastructure communication systems and a human-machine interface.

Today's vehicles are already equipped with a variety of warning and assistance systems, and the utilisation of various automated systems such as Adaptive Cruise Control (ACC), Lane 
Departure Warning Systems (LDWS) and Intelligent Speed Adaptation (ISA) has meant that more and more aspects of the driving task are now controlled by the vehicle. As automation in these vehicles increases, the role of the driver moves from one of an operator to a system supervisor (Bainbridge, 1987). This change in the driver's responsibilities can result in an entirely new set of human factors issues, including the loss of skill (Stanton \& Marsden, 1996), as well as sudden and unwelcome changes in workload, which can lead to 'automation surprises' (Woods et. al., 1994). Increased automation can also result in a loss of situation awareness (Milewski \& Lewis, 1999; Miller \& Parasuraman, 2007) as well as concerns about too little or too much trust in the system (Moray, Inagaki \& Itoh, 2000) a factor which is highly influenced by the system's reliability.

In Europe, the aspirations to increase road safety, allow better management of the road network and reduce fuel consumption have all been partly linked to the introduction of a greater number of advanced technology and automation systems within vehicles and the road transport structure. However, there is still little known about drivers' ability to transfer control to and (re)gain control back from these systems in a fully or highly automated driving scenario. In addition, the effect of this high or full automation of the driving task on driver behaviour and road safety is largely unknown. Many of the assumptions about how automation might influence driver behaviour are based on the work conducted in aviation on pilots (Hancock \& Parasuraman, 1992), perhaps with the exception of studies on the effect of ACC on driver behaviour (Seppelt \& Lee, 2007; Stanton \& Young, 1998).

The objective of the study reported here was to investigate some of the differences in driver behaviour during manual driving, with that of an automated driving scenario. Specifically, this study considered whether and how participants' awareness and understanding of the behaviour of surrounding traffic was influenced by a highly automated driving condition. A particular aim of the experiment was to establish whether drivers had good situation awareness of the driving task in a highly automated driving condition and whether they were able to understand the functionality of the vehicle and its automated system, as well as appreciating how these systems interact with other traffic. This was investigated by comparing driver behaviour in a number of critical situations when the car was driven manually and when driving was automated.

\section{METHOD}

\section{Participants}

Forty participants were recruited for this study (20 female, 20 male). However, since one of the female participants suffered from simulator sickness, results from 39 drivers are reported here. Drivers aged in range between 23 and 63 years (mean $=41$ years), and were all familiar with using the University of Leeds Driving Simulator (UoLDS).

\section{Design and Procedure}

The experiments were conducted in the UoLDS. A within-subjects design was used, with all participants driving one section of urban road which contained two zones in a continuous drive. One zone was driven manually, where all manoeuvres were controlled by the driver, whilst in the 
other, driving was controlled by the vehicle's 'automated system'. This automated system consisted of a longitudinal and lateral controller. The longitudinal controller maintained the speed of the vehicle at $40 \mathrm{mph}$ and retained a headway of 2 seconds to a lead car, whilst the lateral controller kept the vehicle in the centre of the lane. The geometry and layout of the two zones was almost identical. However, half of the drivers drove the manual zone before the automated one, whilst this sequence was reversed for the other half of drivers. All drivers were first given the opportunity to drive the simulator and practice using the automated system, turning it on and off at various points in the drive, experiencing the way in which the control of driving was transferred from the driver to the vehicle, and back again. They were also given ample opportunity to become familiar with the in-vehicle interface (Figure 1), which was used to

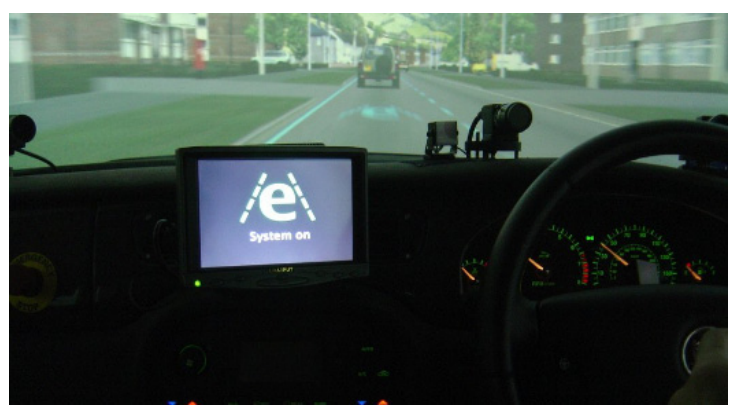

Figure 1. The in-vehicle interface communicate the workings of the automated system to the driver, via audio-visual messages. Once participants were happy with the workings of the vehicle and its automated system, they drove the experimental road, which lasted around 40 minutes, with the driving task controlled by the driver for one half of the drive and by the automated system for the other half of the drive.

Automated driving occurred on an 'eLane' (Flemisch, 2005; Scheiben et al., 2008), which was clearly marked on the road (Figure 2). Participants were alerted about the approach of the eLane via the in-vehicle interface as well as a sign which appeared to the side of the road, $50 \mathrm{~m}$ before the start of the eLane. Upon entering the eLane, drivers were required to transfer control of driving to the vehicle's automated system, by pressing the on/off button on the steering wheel. Once the interface confirmed that the system was active (audio-visual message), drivers took their hands off the steering wheel and their foot off the accelerator pedal and effectively watched the vehicle being driven for them. They were, however, asked to continue monitoring the driving task at all times. If, for whatever reason, drivers wished to regain control of the vehicle within the eLane, they could turn the automated system off by moving the steering wheel, pressing the on/off button on the steering wheel or depressing the brake pedal.

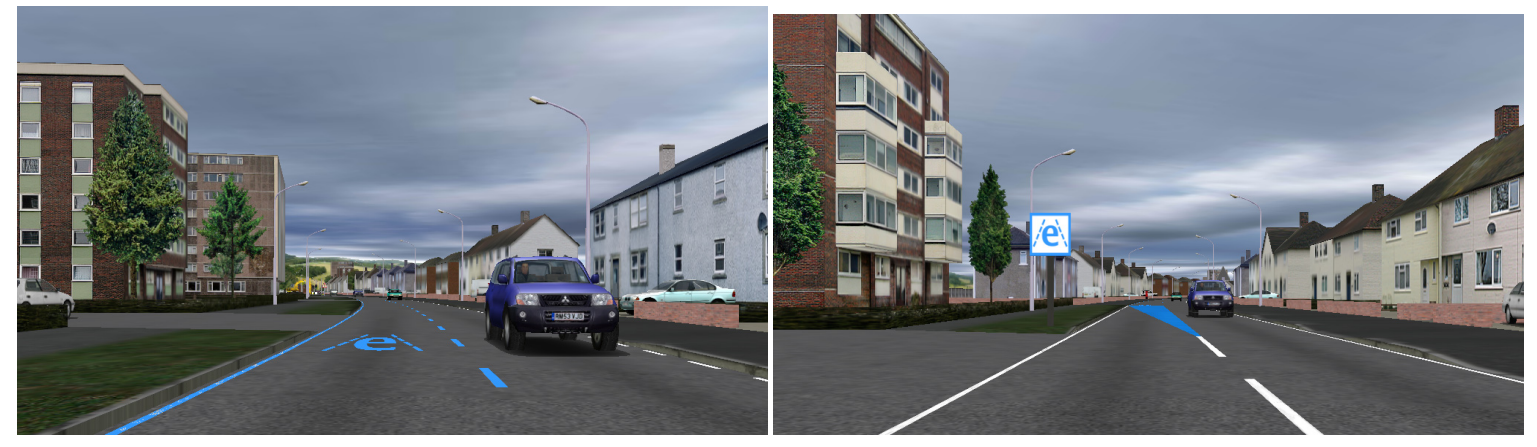

Figure 2. The road markings (left) and sign (right) used to denote the eLane

To allow maximum data collection, most of the drive involved a car following task, where at various points in the drive, the lead car decelerated between $0.5 \mathrm{~m} / \mathrm{s}^{2}$ and $2 \mathrm{~m} / \mathrm{s}^{2}$ in response to a series of non-critical longitudinal events. These included traffic lights changing to red and 
oncoming traffic crossing the lead vehicle's path to enter side roads. In addition to these noncritical events, three longitudinal and one lateral critical event were introduced for both the manual and automated driving zones. For the three longitudinal events, the lead car had to decelerate at a rate of $6 \mathrm{~m} / \mathrm{s}^{2}$, a rate which could not be handled by the automated system. For the lateral event, a vehicle was partly blocking the lane but there was no lead car present. An auditory alarm was used to warn drivers about an impending collision, for all critical events, prompting them to regain control of the driving task from the vehicle's automated system. The same longitudinal and lateral critical events were used for both driving zones; although their order was changed between the two zones to reduce any learning effects (see Table 1). All of the critical events were visible to the simulator driver around 3 seconds before the lead vehicle's brake lights came on. This allowed the driver to anticipate the impending event and respond appropriately, which usually involved braking to avoid a collision. The drivers then transferred control back to the automated system once they had dealt with the critical events.

Drivers' response to these events in the manual road was compared to their reaction in the automated driving event. Therefore, this design examined how drivers' ability to anticipate an impending collision and regain control of driving was affected by an automated driving scenario. At the end of the experiment, drivers were asked to evaluate the automated system and the invehicle interface, using an adapted version of the AIDE-HMI questionnaire.

Table 1. A description of the critical events

\begin{tabular}{l|l|l} 
Event type & Event Description & Event name \\
\hline Longitudinal & $\begin{array}{l}\text { A vehicle emerged from a side road and joined the experimental } \\
\text { road, driving in front of the lead vehicle. }\end{array}$ & Emerger from left \\
\hline Longitudinal & $\begin{array}{l}\text { An oncoming vehicle turned right to enter a side road, crossing the } \\
\text { path of the lead vehicle }\end{array}$ & $\begin{array}{l}\text { Oncomer turns } \\
\text { across }\end{array}$ \\
\hline Longitudinal & $\begin{array}{l}\text { A set of traffic lights changed to red as they were approached by } \\
\text { the lead car }\end{array}$ & Traffic lights \\
\hline Lateral & The road was partly blocked by a parked car or a reversing lorry & Parked car
\end{tabular}

\section{RESULTS}

\section{Vehicle based measures}

To study the effect of automation on driving behaviour, drivers' response to the four critical events in the manual condition was compared to that in automated driving. For the longitudinal critical events, a 2 (DRIVE: manual, automated) by 3 (EVENT: emerger from left, traffic lights, oncoming turns across) repeated measures analysis of variance (ANOVA) was conducted on minimum time to contact and minimum time headway with the lead car, as well as drivers' 'anticipation' of the driving events. This 'anticipation' was measured as the difference in time between the lead car's brake lights coming into sight and when drivers depressed their brake pedal. Each critical longitudinal event was choreographed to allow at least $3 \mathrm{~s}$ for the participants to predict the impending deceleration of the lead vehicle. Therefore, it was assumed that if the driver braked before the lead car, they had a better anticipation of the unfolding events, and a negative value was achieved for anticipation. Similarly, if drivers braked after the lead car, they had less of an anticipation of the emerging events. For the lateral events, a comparison was made 
between drivers' time to contact with the parked car in the road during the manual and automated driving events.

Drivers' minimum time headway to the lead car was found to be significantly longer during the manual condition, compared to the automated driving condition $(\mathrm{F}(1,38)=60.47, p<.0001)$. The ANOVA also showed a significant effect of event, with a significantly longer headway on approach to the traffic light event, compared to the other two longitudinal events $(\mathrm{F}(2,76)=$ $11.20, p<.0001)$. A significant interaction between Drive and Event was also seen here, with the longest minimum headway on approach to the traffic light event in the manual driving condition and the shortest in the automated condition when an emerging vehicle joined the experimental road (see Figure 3).

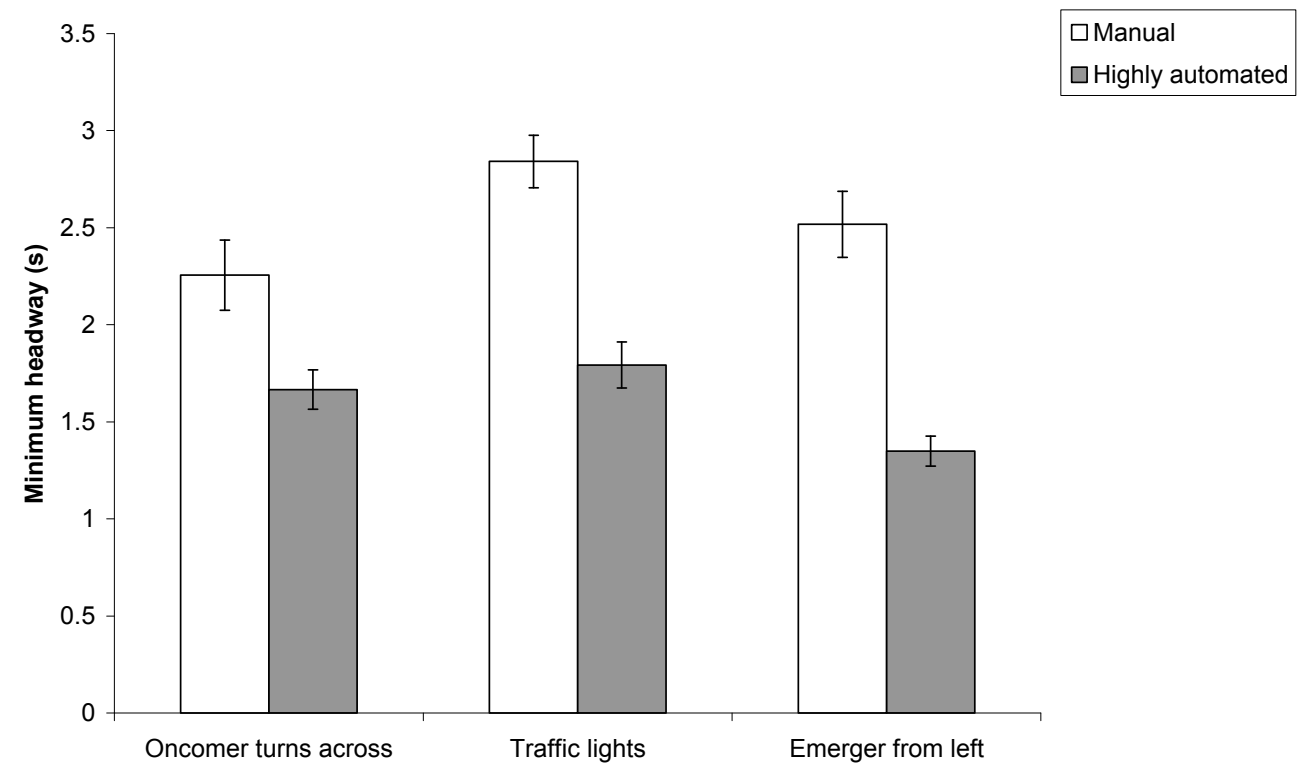

Figure 3. Drivers' minimum headway to the lead car during the manual and automated driving events

Minimum time to contact with the lead car was also found to be significantly different in the manual drive, compared to the automated drive $(F(1,38)=13.77, p=.001)$, with much smaller values seen for the latter condition (1.82 seconds versus 1.44 seconds respectively). There was not a main effect of Event but there was a significant interaction between Event and Drive, where minimum contact to the lead car was particularly short when an emerging vehicle joined the experimental road during the automated drive.

Drivers' anticipation of the three longitudinal events was found to be much less in the automated driving condition, with drivers braking just 0.4 seconds after the lead car braked in the manual condition, compared to 1.90 seconds after the lead car braked in the automated condition $(\mathrm{F}(1,38)=212.83, \mathrm{p}<.0001)$. There was also a significant effect of Event $(\mathrm{F}(2,76)=85.21, \mathrm{p}$ $<.0001)$, where drivers' anticipation of the traffic lights event was found to be the best. The ANOVA also showed a significant interaction between Drive and Event $(\mathrm{F}(2,76)=49.36, \mathrm{p}<$ .0001) with a significantly better anticipation of the traffic lights event by drivers in the manual condition. Results showed that just over half of the drivers (20 out of 39) braked in response to 
the traffic light event after they heard the auditory warning of the automated system. This figure rose to 37 for the 'emerger from left' event, suggesting it was the most difficult longitudinal event for drivers to anticipate.

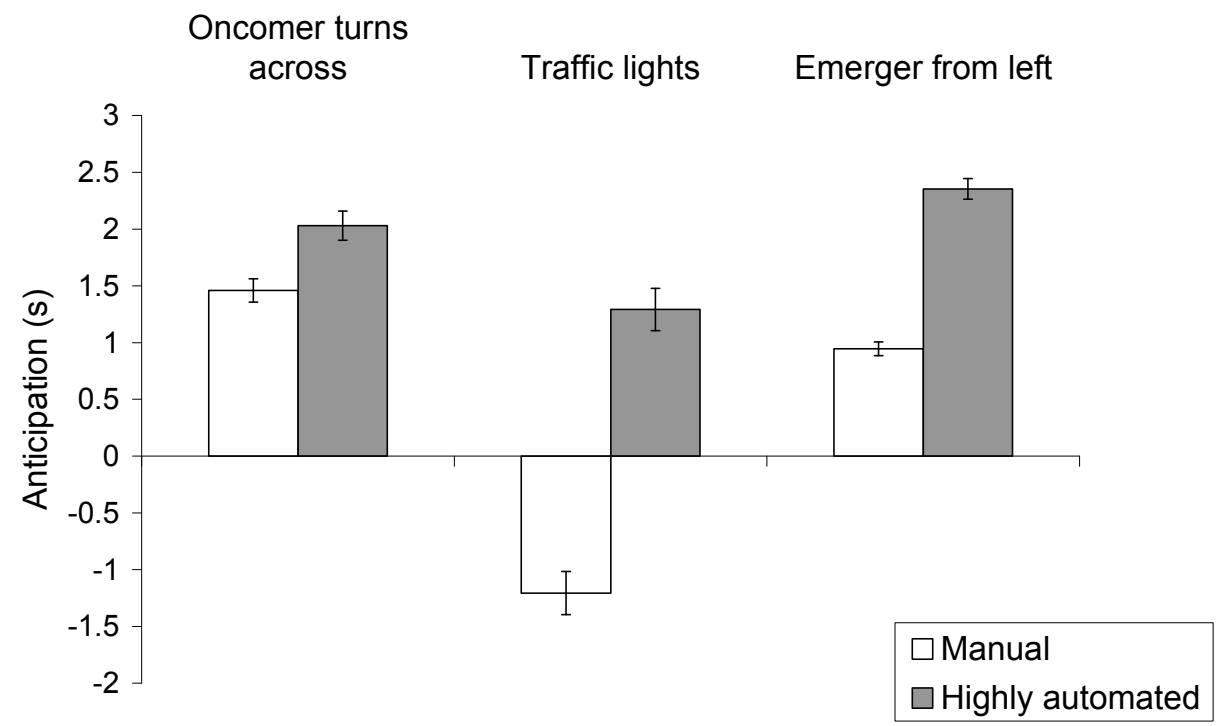

Figure 4. Drivers' anticipation of the three longitudinal events during manual and automated driving

For the lateral critical event, when drivers approached the parked car, all but one driver stopped for a gap in the oncoming traffic before overtaking this obstacle. The average time to contact with the parked car was found to be 4.66 seconds in the manual driving condition ( $\mathrm{SD}=3.55$ seconds), reducing to 2.62 seconds during the automated drive $(\mathrm{SD}=0.89)$. This difference was found to be statistically significant $(\mathrm{F}(1,38)=66.49, p<.001)$. Results also showed that when driving in the highly automated condition, 28 of the 38 drivers braked after the collision warning alarm was emitted.

\section{Subjective measures}

One driver failed to complete the AIDE-HMI questionnaire, therefore the subjective evaluation of 38 drivers is summarised here. The majority of drivers were quite positive about the automated system and the in-vehicle interface, with 31 out of 38 drivers judging the system to be easy to use, whilst 35 drivers thought that it was easy to remember the system's operation. Twenty drivers found the system to be reliable, although only 3 out of 38 drivers thought the system helped them react more quickly to dangerous situations. The majority of drivers thought the warning system, visual interface and vocal messages used by the interface were comprehensible $(35,36$ and 34 out of 38 respectively), whilst just over half of the drivers thought they drove better with the system. 


\section{CONCLUSIONS}

The experiments described here were designed to investigate driver behaviour to a number of critical events when participants drove in a manual mode, compared to when driving was highly automated.

Results showed that, compared to automated driving, drivers' response to all critical events was much faster in the manual driving condition. During the longitudinal critical events, drivers' time to contact and minimum headway with the lead vehicle were found to be much shorter in the automated driving condition, with values significantly less than when driving was controlled manually. In addition, drivers' anticipation of the critical events, which was their ability to predict and understand the behaviour of traffic during such events, was much slower in the automated driving condition, compared to when driving was manual.

Results also showed a difference between the three longitudinal events, where drivers' were able to anticipate the changing lights and respond quickly and appropriately to this event, compared to the other two events. In contrast, understanding the behaviour of the 'emerger from left' event was shown to be most difficult for drivers, with much shorter response times to this critical event during both the manual and automated driving conditions. This was also confirmed by considering drivers' brake response time with respect to the auditory alarm, which showed that 37 out of 39 drivers braked after they had heard the alarm during this event.

The fact that the majority of drivers braked after they heard the auditory alarm during all critical events in the automated driving condition suggests that safety was compromised during this driving condition, when compared to manual driving. However, further experiments are required to establish whether this observed behaviour was because drivers trusted the automated system (perhaps too much?) and deliberately avoided braking until after they heard the alarm, or because the automated driving condition resulted in driver underload, reducing drivers' awareness and understanding of the unfolding traffic events, with the alarm serving as a reminder and bringing their attention back to the driving task.

Overall, drivers provided a positive subjective evaluation of the automated system and in-vehicle interface, which suggests that any differences between the manual and automated driving cannot be due to confusions about the workings of the automated system.

To summarise, one of the human factors challenges associated with highly automated driving is highlighted by this study, which shows that whilst increasing automation in the driving task might be coupled with a more efficient road network and reduced pollution, road safety can only be assured if measures are taken to ensure that the driver remains in the loop at all times and is able to (re)gain control of the driving task quickly and appropriately during unforeseen critical situations. 


\section{ACKNOWLEDGEMENTS}

The authors would like to thank the European Commission for funding this research. The hard work of Anthony Horrobin in generating the driving simulator scenarios is also very much appreciated.

\section{REFERENCES}

AIDE HMI questionnaire, http://www.aide-eu.org/contact.html

Bainbridge, L. (1983). Ironies of automation. Automatica, 19, 775-779.

Flemisch, F.(2005). DLR's Potential Contribution: eLane as a Missing Piece of the CityMobil Automation Concept, Proposal to the CityMobil Preparatory Committee, January 2005.

Hancock, P.A. \& Parasuraman, R. (1992). Human Factors and safety in the design of Intelligent Vehicle-Highway Systems (IVHS). Journal of Safety Research, 23, 181-198.

Milewski, A., \& Lewis, S. (1999). When people delegate (Tech.Memorandum). Murray Hill, NJ: AT\&T Laboratories.

Moray, N., Inagaki, T., \& Itoh, M. (2000). Situation adaptive automation, trust and selfconfidence in fault management of time-critical tasks. Journal of Experimental Psychology: Applied, 6, 44-58.

Schieben, A., Flemisch, F., Marten, M., Wilschut, E., Rambaldini,A., Toffetti, A., Turi, G., Arduino, C., Merat, N. \& Jamson, A.H. (2008). Test results of HMI in use on cars and with simulators, Deliverable 3.2.2., EU Project CityMobil. www.citymobil-project.eu

Seppelt, B.D. \& Lee, J.D. (2007). Making adaptive cruise control (ACC) limits visible. International Journal of Human Computer Studies, 65, 192-205.

Stanton, N.A. \& Marsden, P. (1996). From fly-by-wire to drive-by-wire: Safety implications of automation in vehicles. Safety Science, 24, 35-49.

Stanton, N.A. \& Young, M.S. (1998). Vehicle automation and driving performance. Ergonomics, 41, 1014-1028.

Woods, D.D., Johannesen, L.J., Cook, R.I., \& Sarter, N.B. (1994). Behind human error: cognitive systems, computers, and hindsight. Wright Patterson Air Force Base, Dayton, OH: CSERIAC. 